\title{
MAYORES CUIDANDO MAYORES: SUS PERCEPCIONES DESDE UNA MIRADA INTEGRAL
}

SENIORS CARING FOR SENIORS: THEIR PERCEPTIONS FROM A COMPREHENSIVE POINT OF VIEW

\section{ANCIÃOS CUIDANDO ANCIÃOS: SUAS PERCEPÇÕES DE UM OLHAR COMPLETAMENTE}

\section{Mirliana Ramírez-Pereira}

Universidad de Chile. Santiago, Chile.

mirliana@uchile.cl

ORCID: 0000-0003-1439-4162

\section{Roselyn Aguilera Morales}

Universidad de Chile. Santiago, Chile.

ORCID: 0000-0001-8162-5624

\section{Carla Salamanca Ferrada}

Universidad de Chile. Santiago, Chile.

ORCID: 0000-0003-3531-2891

\section{Catalina Salgado Cuervo}

Universidad de Chile. Santiago, Chile.

ORCID: 0000-0001-8332-6513

\section{Constanza San Martín Silva}

Universidad de Chile. Santiago, Chile.

ORCID: 0000-0003-4023-2853

\section{Claudia Segura Díaz}

Universidad de Chile. Santiago, Chile.

ORCID: 0000-0001-8141-3664

DOI: https://doi.org/10.22235/ech.v7i2.1653

Recibido: 13/12/2017

Aceptado: 22/06/2018 
Resumen: El envejecimiento es un proceso que conlleva múltiples cambios en el ser humano, generando que algunos adultos mayores se encuentren en situación de dependencia y requieran un cuidador. Este estudio cualitativo, con enfoque metodológico de estudio de caso, tiene por objetivo conocer las percepciones de adultos mayores que son cuidadores de otro adulto mayor con dependencia severa. Se realizaron entrevistas en profundidad empleando análisis de contenido y se definieron las siguientes categorías de investigación: motivaciones, dificultades, emociones del cuidador, postergación por el bien del otro, redes de apoyo familiares, emociones asociadas al diagnóstico, religión, institucionalización, expectativas de cuidado de enfermería, acceso a beneficios del estado, y muerte. Los resultados muestran que la mayoría de los cuidadores son mujeres, sin apoyo familiar, que tienen un vínculo sentimental con la persona cuidada, y que sus motivaciones para desempeñar esta labor son el cariño y la obligación. Se observó postergación por parte del cuidador dedicándose por completo a esta labor, abandonando actividades ajenas al cuidado y experimentando sobrecarga acompañada de limitaciones económicas debido a los bajos ingresos que reciben.

Palabras claves: Investigación cualitativa, enfermería, anciano, percepción, cuidadores, dependencia.

\begin{abstract}
Aging is a process that involves multiple changes in the human being, forcing some elderly people in a situation of dependency to require a caregiver. This is a qualitative study with a methodological approach of case study, aiming to know the perceptions of older people who are caregivers of another elderly people with severe dependence. In-depth interviews were conducted using content analysis. The following categories were defined: motivations, difficulties, caregiver emotions, self postponement for the sake of the other, family support, emotions associated with the diagnosis, religion, institutionalization, nursing care expectations, access to state benefits, and death. The results show that most caregivers are women, without family support, who have a sentimental bond with the person cared for, and that their motivations to perform this task where affection and obligation. Self postponement was observed; the caregiver devots completely to this task, abandoning other activities and experiencing burden accompanied by economic limitations due to the low income they receive.
\end{abstract}

Keywords: Qualitative research, nursing, aged, perception, caregiver, elderly, nursing, dependence.

Resumo: $O$ envelhecimento é um processo que envolve múltiplas mudanças no ser humano, gerando algumas pessoas idosas em uma situação de dependência, exigindo um cuidador. Este estudo qualitativo, com abordagem metodológica de estudo de caso, visa conhecer as percepções de idosos que são cuidadores de outra pessoa idosa com dependência severa. Foram realizadas entrevistas em profundidade utilizando análise de conteúdo e foram definidas as seguintes categorias de pesquisa: motivações, dificuldades, emoções do cuidador, adiamento para o bem do outro, redes de apoio familiar, emoções associadas ao diagnóstico, religião, institucionalização, expectativas de atendimento de enfermagem, acesso a benefícios estatais e morte. Os resultados mostram que a maioria dos 
cuidadores são mulheres, sem apoio familiar, que têm vínculo sentimental com a pessoa atendida e que suas motivações para realizar este trabalho são carinho e obrigação. $\mathrm{O}$ adiamento foi observado por parte do cuidador, dedicando-se completamente a essa tarefa, abandonando atividades que não sejam cuidados e experimentando sobrecarga, acompanhada de limitações econômicas devido à baixa renda que recebem.

Palavras-chave: Pesquisa qualitativa, enfermagem, percepção, cuidadores, idosos, enfermagem, dependencia.

\section{INTRODUCCIÓN}

El aumento del índice de envejecimiento en Chile ha constituido durante las últimas décadas un cambio social importante que se relaciona con la incorporación de un nuevo grupo etario con roles y características que se mantienen en constante variación debido a la reestructuración social (1). El Instituto Nacional de Estadísticas (INE) señala que a nivel nacional, en 2014 el número de adultos mayores (personas con 60 años y más) era de 2,6 millones, cifra que representa un $15 \%$ de la población total y que supera al $11 \%$ de 2002 (2). A partir de estos datos el Servicio Nacional del Adulto Mayor (SENAMA) apunta que Chile también ha formado parte de este cambio demográfico, posicionándose como el segundo país más envejecido de Latinoamérica (3).

En este contexto, el envejecimiento plantea desafíos para los sistemas de salud, la economía y la política, pues trae consigo inequidades y problemáticas de una población que ha sufrido las consecuencias de una historia de vida de trabajo y sacrificio. Esto ha generado la necesidad de implementar iniciativas que vayan en beneficio de esta población, instaurando a nivel estatal políticas públicas como el Programa Chile Cuida.

El panorama actual para las personas mayores se enlaza con las enfermedades crónicas no transmisibles y riesgo socioeconómico; estos factores pueden deteriorar la calidad de vida de los adultos mayores y afectar a sus familias (4).

Se ha estudiado la necesidad de requerir un cuidador permanente para satisfacer las necesidades de los adultos mayores y se han descrito las características de las personas que cumplen este rol, quienes son principalmente esposas e hijas de la persona receptora de cuidados. Conociendo que la población envejece y que, por lo tanto, la edad pasa de igual manera para todos los integrantes de cada núcleo familiar, este estudio plantea la necesidad de estudiar a personas que siendo mayores cuidan de otros mayores, para, de esa forma, acercarnos a una esfera de su día a día que nos permita conocer de qué manera desempeñan esta labor y cómo influye en su calidad de vida desde su propia percepción, considerando como fin último contribuir a la implementación de estrategias que mejoren las condiciones de vida de las personas mayores del país.

\section{METODOLOGÍA}

Esta investigación corresponde a un estudio de carácter cualitativo, realizado en un Centro de Salud Familiar del sector norte de la ciudad de Santiago. El objetivo fue conocer las percepciones que tienen los adultos mayores que cuidan a otros adultos mayores con dependencia severa respecto a su calidad de vida. 
El paradigma empleado para esta investigación es el post positivista, en el cual se plantea que la realidad existe, pero no puede ser completamente aprehendida (5). Para esta investigación se utilizaron los criterios de rigor desarrollados por Guba y Lincoln: credibilidad, transferibilidad, dependencia y confirmabilidad (6), los cuales se cumplieron por medio de las notas de campo, transcripción de las entrevistas con revisión del entrevistado y triangulación de investigadores.

Por su parte, el enfoque teórico metodológico a utilizar fue el estudio de caso, empleado para investigaciones a pequeña escala en un marco de tiempo y recursos acotados, adecuándose al contexto del estudio con la finalidad de conocer situaciones únicas que merecen interés de investigación permitiendo un acercamiento al día a día del adulto mayor (7).

La técnica de recolección de datos utilizada en este estudio fue la entrevista en profundidad, para las que se definieron previamente las siguientes unidades de significado: motivaciones, dificultades, emociones del cuidador, postergación por el bien del otro y redes de apoyo familiares, considerándose la posibilidad de obtener unidades de significado emergentes.

Estas entrevistas fueron transcritas sin edición por parte de las propias autoras y posteriormente analizadas mediante la metodología de análisis de contenido. Según Mayring el análisis de contenido permite descubrir la significación de un mensaje, clasificando y/o codificando los diversos elementos en unidades definidas, con el propósito de identificar de forma objetiva y sistemática su sentido, logrando su interpretación (8).

Para la selección de los participantes se utilizó el método de muestreo por conveniencia, el que permitió seleccionar aquellos casos accesibles que aceptaran ser incluidos en el estudio. Esto está fundamentado en la conveniente accesibilidad y proximidad de los participantes (9). Así también, para este estudio se consideró el criterio de saturación teórica, el que permite dar fin a la recolección de datos una vez que la información obtenida comienza a hacerse reiterativa, sin aportar nuevos hallazgos (10), lo que se alcanzó con el relato de seis participantes.

Los participantes de este estudio fueron seleccionados considerando los siguientes criterios de inclusión: ser adulto mayor (60 años o más), voluntarios, de ambos géneros, con ingresos económicos inferiores a USD 412 mensuales (considerando que este valor corresponde al ingreso mínimo mensual en Chile), que se encuentren hace dos años o más al cuidado de otro adulto mayor en situación de dependencia severa según índice de Barthel (11) y que firmen el consentimiento informado.

En esta investigación se desarrollaron los principios éticos de valor social o científico, la validez científica, selección equitativa de las personas, proporción favorable del riesgobeneficio, evaluación independiente, consentimiento informado y respeto a las personas inscritas (12). Considerando que es una investigación cualitativa, a los criterios antes mencionados se sumó el de condiciones de diálogo auténtico, lo que se consiguió con la presentación de los resultados a los participantes (13). 
Esta investigación fue aprobada por el comité de ética de Investigación en Seres Humanos de la Facultad de Medicina de la Universidad de Chile con el Acta de aprobación del proyecto $\mathrm{N}^{\circ} 115-2017$ y por el comité de ética del Centro de Salud Familiar. Los participantes de esta investigación firmaron el consentimiento informado previo a la realización de las entrevistas.

\section{RESULTADOS Y ANÁLISIS}

A continuación, se presentan los resultados y el análisis de las entrevistas realizadas. En la siguiente tabla se resumen algunos antecedentes de los participantes de este estudio.

Tabla 1: Caracterización de los participantes

\begin{tabular}{|c|c|c|c|c|c|c|}
\hline Participante & Edad & Género & $\begin{array}{c}\text { Años } \\
\text { cuidando }\end{array}$ & $\begin{array}{c}\text { Edad de la } \\
\text { persona } \\
\text { cuidada }\end{array}$ & $\begin{array}{c}\text { Género de la } \\
\text { persona } \\
\text { cuidada }\end{array}$ & Parentesco \\
\hline LE & 78 & M & 17 & 78 & F & Esposa \\
\hline JV & 69 & M & 4 & 63 & F & Esposa \\
\hline RG & 73 & F & 4 & 81 & M & Conviviente \\
\hline IP & 68 & F & 3 & 68 & M & Esposo \\
\hline MF & 90 & F & 2 & 92 & M & Esposo \\
\hline SM & 63 & F & 17 & 89 & F & Madre \\
\hline
\end{tabular}

Fuente: Elaboración propia (2017)

A partir del proceso de análisis inductivo y deductivo se construyeron las unidades de significados: "Motivaciones para cuidar"; "Dificultades en el cuidado"; "Emociones del cuidador"; "Género", “Acceso a beneficios del Estado”, "Creencias religiosas", "Postergación como persona", "Redes de apoyo familiar; "Expectativas de cuidado de enfermería"; "Miedo a la institucionalización"; y "La muerte".

A continuación, se describen las unidades de significados antes mencionadas:

En la unidad de significado "Motivaciones para cuidar" los entrevistados manifiestan que las razones que motivan el cuidado son el cumplimiento de un deber, acompañado de la falta de opciones y el amor por el familiar que se encuentra postrado. Los participantes consideran el cuidado como una lucha continua por falta de opciones dadas principalmente por la falta de redes de apoyo.

"Yo creo que las dos cosas, porque si no fuera por el amor y el cariño no habría luchado tanto (toma una pausa y se emociona) y bueno, tengo que seguir luchando porque no 
queda de otra opción, no es que yo quiera porque sí, sino que no me queda de otra opción, es asi de simple, Es un deber..." (J.V.)

La categoría "Dificultades en el cuidado" hace referencia a los problemas y limitaciones que enfrentan los cuidadores en el día a día. Dentro de éstos, los entrevistados señalan que los principales problemas que presentan son de tipo económico y que las limitaciones más importantes se asocian a la disminución del tiempo disponible para realizar otro tipo de actividades que no se relacionen con el cuidado. Se puede apreciar que los participantes de este estudio perciben dos tipos de pensiones: los adultos mayores que impusieron durante su etapa laboral reciben pensiones mediante las administradoras de fondos de pensiones, instituciones financieras privadas que administran los dineros que son depositados de manera mensual por los trabajadores dependientes del país y que posteriormente son fraccionados y entregados cada mes a los adultos mayores cuando estos finalizan su etapa laboral. Por otro lado, los adultos mayores que no tienen derecho a percibir una pensión bajo algún régimen previsional y cumplan los requisitos establecidos por el estado son beneficiarios de pensiones básicas solidarias de vejez. En ambos casos, las pensiones que reciben son precarias, no superan los USD 412 mensuales y con ellas los adultos mayores no logran cubrir todas sus necesidades básicas. Con respecto a las limitaciones en torno a tiempo, el hecho de cuidar a otro dificulta el desarrollo de una vida normal, por lo que el cuidador debe dedicarse por completo al ser cuidado, dejando de lado sus temas personales.

"Y a veces nos falla el dinero también, sí... porque también uno saca una miseria de sueldo...que no te dejan desarrollarte como persona, o sea, no puedes hacer nada.” (S.M.)

La unidad de significado "Emociones del cuidador" hace alusión a las emociones que emergen en el cuidador asociadas a la labor que desempeña. Se observa que predominan la tristeza y la angustia, las que se expresan de forma más frecuente a través del llanto, que nace de manera natural a medida que se desarrollan las entrevistas y que se relaciona mayoritariamente con el deterioro físico que experimenta el ser cuidado.

"He llorado mucho, mucho. De verlo tan flaquito, si está tan flaquito, con los huesitos parados" (M.F.)

Los entrevistados destacan que en el momento de conocer el diagnóstico de su ser querido su primera respuesta fue la negación en torno a la condición de salud que éste experimenta.

"Siempre el no creer, no creer el cáncer, yo le decía: no tienes nada, te van a sacar esto (refiriéndose al tumor) y vas a quedar bien" (S.M.)

La unidad de significado "Género" muestra la visión que tienen los entrevistados frente a los roles de género impuestos por la sociedad en su rol de cuidador. Es posible apreciar la marcada presencia de estos roles tradicionales de género en el discurso de los entrevistados, los cuales señalan que la mujer es la encargada del cuidado y el hombre es el proveedor y sostenedor familiar.

"Es dificil porque los hombres no estamos preparados para cuidar. O sea, la mujer tiene un don especial porque la mujer desde chiquitita parece, nacen con ese don hasta toda su 
vida. Ven a los papás, a los hijos, van a trabajar, trabajan en la casa, si uno de repente que viene a hacer eso, cuesta, porque uno, uno va a trabajar, trae la plata, se va a la cancha a jugar futbol. Ella está en la casa." (J.V.)

Al invertir los roles, los cuidadores mencionan que les fue difícil asumir dicha posición debido a que las tareas que realizan diariamente son socioculturalmente asociadas al género femenino.

"Yo cocino, yo hago todo acá. Tengo que lavar. Como una dueña de casa." (J.V.)

La categoría "Acceso a beneficios del Estado" hace referencia a la apreciación que tienen los cuidadores respecto al acceso a beneficios estatales, que apoyan la tarea de cuidar. Los entrevistados señalan que existe una oferta escasa de servicios a su disposición, los cuales no son bien percibidos en un principio, pero que luego de conocerlos valoran la ayuda que se les presta. La desconfianza inicial se produce porque los ven como desconocidos, que no forman parte de su núcleo familiar y que entran a su casa.

"Nos ha pasado con las del Chile Cuida que ella al principio no quería que vinieran a cuidarla, hacer la limpieza de la casa o a cocinar, pero en ese sentido nunca hubo problema porque siempre el almuerzo estaba... más bien llegaban a conversar" (L.E.).

Sin embargo, los cuidadores destacan que se han visto beneficiados con la ayuda proporcionada por el programa "Chile Cuida".

"Felizmente, yo digo felizmente, que hay un programa, no sé si ustedes lo han oído hablar siquiera...Chile Cuida”. (S.M.)

En la unidad de significado "Creencias Religiosas" se desprende de los discursos un fuerte arraigo a la religión que existe en la sociedad chilena. En estos, se destaca a Dios como un ente que determina sus vidas, que los acompaña, fortalece y consuela en los momentos difíciles.

"Sólo Dios va a saber qué es lo que me tiene en el destino, cierto, entonces hay que esperar no más”. (J.V.)

"Uno sin fe no se hace nada. Es un apoyo, la fe que uno tiene, porque uno tiene alguien que uno se conversa con alguien y se llena de energía nueva porque si no, uno no sale..." (M.F.)

La categoría "Postergación como persona" hace referencia a las actividades que los cuidadores han dejado de realizar desde que desempeñan este rol, el deterioro del autocuidado que experimentan y la importancia que otorgan a su familiar. Se observa que la prioridad es satisfacer las necesidades básicas de la vida diaria del ser querido (que no puede realizarlas por sí mismo), lo que muchas veces limita el ejercicio del autocuidado por parte del propio cuidador. Si bien en algunos casos se identifica adherencia a la realización de actividades recreativas, la mayoría posterga sus propias necesidades y desarrollo personal por el bien de la persona cuidada. 
"Mi prioridad no es mi prioridad en este minuto tampoco, mis prioridades son estar con la mamá, terminar con la mamá”. (S.M.)

"De partida, tuve que dejar de trabajar para cuidarla... una de las primeras causas. Y la segunda de que la enfermedad que ella tiene es progresiva, no tiene remedio, entonces me tuve que quedar con cuidarla no más." (J.V.)

Respecto a las "Redes de apoyo familiar" se recogen las opiniones de los cuidadores respecto al apoyo en el cuidado recibido por su familia. De los relatos se desprende la ausencia de redes de apoyo familiares, observándose la desvinculación de los miembros del núcleo familiar respecto a la labor de cuidar. De esta manera, la responsabilidad recae completamente en el adulto mayor que ejerce el rol de cuidador principal, quien debe cumplir esta labor ya que no hay ninguna persona más que quiera asumirla.

"Tú sabes que en estas situaciones siempre hay uno que se hace más cargo que todos los demás, que todos los demás hacen vista gorda de todo y todos se olvidan de que fuimos cuatro, o sea que fuimos cinco, sin contar mi hermana que falleció, ellos se olvidaron de que también fueron criados por una mamá, no tan solo uno”. (R.G.)

En la categoría "Expectativas del cuidado de Enfermería” los cuidadores manifiestan qué es lo que esperan de la atención brindada por parte de los profesionales de Enfermería. En los testimonios se destaca que lo esperado por parte de los entrevistados es principalmente un trato ameno, con cariño y donde prime el respeto, esperando que los profesionales de Enfermería ejerzan su rol desde la vocación, buscando siempre el bienestar del adulto mayor.

"Con cariño, no con que "ah, este viejo”, siempre que sea con un cariño, con un don que tengan de cuidar. No faltando el respeto, tener respeto con todo.” (I.P.)

La categoría "Miedo a la Institucionalización" recoge las opiniones que tienen los cuidadores respecto a la idea de institucionalizar a su ser querido. Se evidencia una marcada oposición por parte de los adultos mayores entrevistados al hecho de dejar que la persona cuidada sea institucionalizada, prefiriendo ejercer el cuidado ellos mismos a pesar de que los demás miembros de la familia no estén presentes.

"No, no, no, no, eso sí que no, no. Nunca he pensado en eso, ni nadie de mi familia ha pensado "lo vamos a llevar, porque no te deja tranquila", no. Déjenme a mí, si ellos no quieren estar, pero yo estoy aquí." (J.V.)

En la categoría "La muerte" los cuidadores señalan sus apreciaciones frente a la muerte y las emociones que emanan cuando se piensa en el término de la vida. Siendo la vejez la cúlmine del ser humano, la muerte se convierte en un elemento latente en los relatos de todos los entrevistados. Se destaca el fuerte impacto que genera a nivel emocional este tema, causando angustia y temor en los cuidadores. 
"Sé que la tengo que dejar partir con mi dolor fuerte que siento, sé que la tengo que dejar partir, tal vez yo soy egoísta en llorar, pero es algo de uno, tú no puedes evitarlo. Es un proceso demasiado fuerte, es un proceso demasiado duro". (I.P.)

\section{DISCUSIÓN}

Los discursos de los participantes muestran estereotipos de cuidado tradicionalmente asociados al género femenino, lo que se condice con la investigación realizada por Flores, que muestra que existe un marcado constructo social que asocia al género femenino con las tareas de cuidado (14). Cuando el hombre asume el rol de cuidador se enfrenta a las desigualdades de género construidas por la sociedad, las cuales se invierten en estas situaciones e impactan en la percepción de los cuidadores, quienes manifiestan en sus relatos que ha sido complejo cumplir con este rol al no encontrarse preparados para asumirlo de manera "natural". En el caso de los hombres entrevistados en este estudio se observa que existe una falta de expresión de las emociones, lo que puede estar asociado a los estereotipos de género, los que empujan a los hombres a mantenerse estoicos frente a la adversidad, mientras que a las mujeres se les permite expresar abiertamente sus emociones (15).

En algunos estudios internacionales se señala que las cuidadoras desempeñan un doble rol, ya que se dedican al cuidado de una persona dependiente y a un trabajo remunerado (16). Sin embargo esta tendencia no se mantiene en las cuidadoras entrevistadas, quienes debido a su edad han abandonado el mundo laboral y se dedican exclusivamente a cuidar, lo que genera que los ingresos que reciben procedan exclusivamente de sus pensiones. Esto es preocupante, considerando la precariedad del sistema de pensiones en Chile, el cual no protege los intereses de los adultos mayores y provee ingresos insuficientes para cubrir sus necesidades básicas, considerando además que la dependencia severa de la persona receptora de cuidados incrementa los gastos económicos del día a día.

La falta de recursos económicos es percibida por los cuidadores como la principal dificultad que se presenta en su labor, lo que impide que puedan destinar ingresos a otras actividades que sean beneficiosas para su calidad de vida. Esto, sumado a las extensas horas dedicadas al cuidado, el difícil acceso a los servicios de salud y la ausencia de apoyo familiar promueven la aparición de sobrecarga en el cuidador, fenómeno transversal a todos los entrevistados en esta investigación, lo que se condice con los estudios revisados (17). Es importante destacar que en la literatura revisada se señala que para que el cuidado de las personas mayores se pueda sobrellevar, se debe distribuir el trabajo en la familia de "forma igualitaria y equitativa", por lo que la tarea del cuidado no debe recaer en solo una persona (14), lo cual contrasta con los relatos de los entrevistados, quienes manifiestan que el cuidado recae únicamente en ellos, lo que agrava la condición de sobrecarga que experimenta el cuidador.

En varios estudios se señala que el cuidado de una persona con dependencia se asocia a efectos negativos para la salud del cuidador, generalmente vinculados al ámbito psicoafectivo como depresión. Así también, los resultados de esta investigación dan cuenta de que esta situación se repite entre los participantes, pues la mayoría de ellos señaló 
percibir un deterioro en su salud luego de cuidar, sobre todo en la esfera de la salud mental. Si bien no se puede establecer una relación causal entre cuidar y sufrir alteraciones de la salud, es importante considerar que dado el contexto en que se ejerce el cuidado, esto podría verse acentuado también con el hecho de que existe un lazo sentimental con la persona receptora de cuidados y que asumen esta responsabilidad sin contar con ayuda de terceros (18).

Respecto a la institucionalización, cabe señalar que estudios realizados en Suecia evidencian que la sobrecarga y la sensación de necesidades personales insatisfechas en los cuidadores se asocian al deseo de éstos de institucionalizar a la persona que cuidan. Esto se contrasta con los hallazgos de esta investigación, los que muestran una marcada oposición por parte de los adultos mayores entrevistados a dejar que el ser cuidado sea institucionalizado, prefiriendo ejercer el cuidado ellos mismos, a pesar de que los demás miembros de la familia no estén presentes (19).

En cuanto al nexo que se puede establecer entre este estudio y la teoría de Duvall del Ciclo Vital Familiar, es posible evidenciar que la mayoría de las familias se encuentra en la octava etapa del ciclo familiar, donde los núcleos familiares adquieren la denominación de "familias ancianas", concluyendo esta etapa con el fallecimiento de ambos cónyuges. De acuerdo a esta fase se tiene como meta el ejercicio de un cuidado mutuo en la vejez, lo cual no se ve reflejado en este estudio, ya que el cuidado es desempeñado de manera unilateral. En relación a las tareas que se deben cumplir en esta fase se destaca el incumplimiento de la regulación entre la dependencia y la independencia, ya que no existe un equilibrio entre estos tópicos en la vida de los adultos mayores entrevistados. Al contrario, es posible apreciar cómo los cuidadores cumplen con la tarea de enfrentar la enfermedad y la muerte próxima de su ser querido, haciendo latente en sus relatos esta temática (20).

Finalmente, de acuerdo con la Teoría de Orem se pueden distinguir dos tipos de cuidado: el que ejerce la persona sobre sí mismo, denominado autocuidado, y el cuidado que es brindado a otra persona que no es capaz de satisfacer sus necesidades básicas por sí mismo (21). En este estudio se puede ver como el autocuidado se ve afectado en la salud del cuidador, no solo en el ámbito físico, sino que también en el ámbito emocional y psicológico, repercutiendo en su salud mental.

\section{CONCLUSIÓN}

Dentro de los hallazgos de esta investigación destacan la tristeza sentida por los cuidadores por el deterioro físico y mental que experimenta su ser querido, manifestándolo a través del llanto a lo largo de sus relatos. Los cuidadores se postergan en beneficio de su ser querido, abandonando actividades ajenas al cuidado y dedicando por completo su tiempo a satisfacer las necesidades de la persona cuidada, experimentando una sobrecarga que impacta en todas las áreas del ser humano. En cuanto a las dificultades, predominan las limitaciones económicas, donde los adultos mayores perciben ingresos a través de sus pensiones que no superan los USD 412 mensuales, con los cuales no logran satisfacer sus necesidades básicas. Esto se acrecienta con la carencia de redes de apoyo familiares, quedando en 
evidencia cómo el cuidador desempeña su labor de manera solitaria, sin recibir ayuda de terceros pertenecientes a su núcleo familiar.

Es en extremo preocupante que, a pesar de que la población está envejeciendo, existan pocas investigaciones respecto a los cuidados brindados por adultos mayores a contemporáneos en condición de dependencia, siendo este estudio pionero en este campo. Ante ello, esperamos que pueda ser el comienzo de más estudios en los cuales se profundice este tema y se pueda trabajar activamente en iniciativas que contribuyan a mejorar la calidad de vida de esta población.

Se sugiere la participación de Enfermería en programas gubernamentales de apoyo al cuidador, como el programa Chile Cuida, ya que la Enfermería es un arte y disciplina dedicada al cuidado del ser humano y por lo tanto debe ser quien lidere este tipo de iniciativas por ser quien posee más conocimiento en el tema. Junto con ello, el incrementar los conocimientos sobre esta temática permite que los profesionales de Enfermería proporcionen cuidados integrales y personalizados, de acuerdo al contexto que engloba la cotidianidad de los cuidadores.

Este estudio proporciona una nueva visión, desde la investigación cualitativa, que permite enriquecer la escasa información que se tiene hasta el momento de esta temática, obtenida a través de investigaciones de tipo cuantitativo.

\section{REFERENCIAS BIBLIOGRÁFICAS}

1. Instituto Nacional de Estadísticas. Actualización de proyecciones de población 20022020 [Internet] 2014 [Citado Mayo 2017] Disponible en: http:/www.ine.cl/canales/sala_prensa/revistaseconomicas/presentaciones/pdf/proyecciones _poblacion_2014.pdf

2. Instituto Nacional de Estadísticas (2014). Actualización de proyecciones de población (2002-2020). Recuperado el 8 de Mayo de 2017, de: http://www.ine.cl/canales/sala_prensa/revistaseconomicas/presentaciones/pdf/proyecciones poblacion_2014.pdf

3. SENAMA (2011) Chile quiere a sus mayores. Recuperado el 7 de abril de 2017, de http://www.senama.cl/filesapp/cuentapublica2011.pdf

4. Ministerio de Salud. Programa nacional de salud de las personas mayores. [Internet] 2014 [Citado Abril 2017] Disponible http://web.minsal.cl/sites/default/files/files/Borrador\%20documento $\% 20$ Programa $\% 20 \mathrm{Naci}$ onal\%20de\%20Personas\%20Adultas\%20Mayores-\%2004-03_14.pdf

5. Ramos CA. Los paradigmas de la investigación científica. Av. Psicol. [Internet] 2015 [Citado May 2017] 23 (1), 9-17. Disponible en: https:/www.researchgate.net/publication/282731622_LOS_PARADIGMAS_DE_LA_INV ESTIGACION_CIENTIFICA_Scientific_research_paradigms 
6. Johnson S. Rasulova S. Qualitative impact evaluation: incorporating authenticity into the assessment of rigour. Journal of Development Effectiveness. 2017; 9 (2) 263-276

7. Murillo J. Estudio de casos. Universidad Autónoma de Madrid. 2002.

8. Mayring P. Qualitative Content Analysis. Forum Qualitative Social Research. 2000; 1 (2). 20.

9. Otzen Tamara, Manterola Carlos. Técnicas de Muestreo sobre una Población a Estudio. Int. J. Morphol. [Internet]. 2017 Mar [citado 2017 Dic 11] ; 35( 1 ): 227-232. Disponible en: $\quad$ http://www.scielo.cl/scielo.php?script=sci_arttext\&pid=S071795022017000100037\&lng=es. http://dx.doi.org/10.4067/S0717-95022017000100037.

10. Arias Valencia MM, Giraldo Mora CV. El rigor científico en la investigación cualitativa. Investigación y Educación en Enfermería [Internet] 2011 [citado Abr 2017] 29(3): 500-514. Disponible en: http://www.redalyc.org/articulo.oa?id=105222406020

11.Ministerio de Salud de Chile. Orientación técnica para la atención de salud de las personas adultas mayores en atención primaria, 2013.

12. Emanuel E. What makes clinical research ethical. JAMA. 2000283 (20): 2701-11.

13. Miguelez B. Investigación social cualitativa y dilemas éticos: de la ética vacía a la ética situada. Empiria.2016; (34): 101-20.

14. Flores E, Rivas R, Seguel F, Nivel de sobrecarga en el desempeño del rol del cuidador familiar de adulto mayor con dependencia severa. Ciencia y enfermería [Internet] 2012 [Citado abril 2017] 18(1), 29-41. Disponible en: https://dx.doi.org/10.4067/S071795532012000100004

15. Gartzia L. Aritzeta A, Balluerka N, Barberá E. Inteligencia emocional y género: mas allá de las diferencias sexuales. Anales de Psicología [Internet]. 2012; [Citado Julio 2018] 28 (2): 567-575. Disponible en: http: //www.redalyc.org/articulo.oa? id=16723135028.

16. Larrañaga I, et al. Impacto del cuidado informal en la salud y la calidad de vida de las personas cuidadoras: análisis de las desigualdades de género. Gaceta sanitaria [Internet] 2008 [Citado junio 2017] 22(5), 443-450. Disponible en: http://www.sciencedirect.com/science/article/pii/S0213911108724191

17. Espinoza K. Principales necesidades de cuidados de enfermería en cuidadores familiares de adultos mayores postrados. Tesis de Enfermería. Universidad Austral de Chile [Internet] $2010 \quad$ [Citado abril 2017] Disponible en: http://cybertesis.uach.cl/tesis/uach/2010/fme.77p/doc/fme.77p.pdf 
18. Estrada M. et al. Dependent relative: Effects on family health. Atención Primaria [Internet] 2017 [Citado Abril 2017] Disponible en: http://dx.doi.org/10.1016/j.aprim.2016.12.007

19. Blusi M, Kristiansen L, Jong M. Exploring the influence of Internet-based caregiver support on experiences of isolation for older spouse caregivers in rural areas: A qualitative interview study. Int J Older People Nurs. 2015 Sep;10(3):211-220.

20. Martin T. Family Development Theory 30 Years Later. J FamTheory Rev. 2018; 10: 49-69

21. Marriner Tomey A, Alligood MR. Modelos y teorías en enfermería. $6^{\circ}$ ed. Barcelona: Elsevier; 2007. 